\title{
Is genetic diversity really higher in large populations? Hans Ellegren
}

Address: Department of Evolutionary Biology, Uppsala University, Norbyvägen 18D, SE-752 36 Uppsala, Sweden. Email: Hans.Ellegren@ebc.uu.se

Published: 21 April 2009

Journal of Biology 2009, 8:4I (doi: I0.1 I86/jbioll 35)

The electronic version of this article is the complete one and can be found online at http://jbiol.com/content/8/4/4I

(c) 2009 BioMed Central Ltd

\begin{abstract}
Analyses of mitochondrial DNA (mtDNA) have challenged the concept that genetic diversity within populations is governed by effective population size and mutation rate. A recent study in BMC Evolutionary Biology shows that variation in the rate of mutation rather than in population size is the main explanation for variations in mtDNA diversity observed among bird species.
\end{abstract}

Students of population genetics learn from their textbooks that levels of genetic diversity are determined by the rate of mutation (the number of new mutations per nucleotide site and generation) and the number of reproducing individuals in the population. The latter is usually termed the effective population size $\left(N_{e}\right)$, and is almost always less than the total population size. These determinants are intuitive, as a higher rate of mutation creates more new genetic variants and a large population is less sensitive to random loss of variants by genetic drift. It therefore came as a surprise when it was reported a few years ago that genetic variability in mitochondrial DNA (mtDNA), one of the most commonly used markers in evolutionary and population genetic analysis, did not seem to correlate with population size when analyzed over broad taxonomic groups. For example, levels of polymorphism were found to be similar for invertebrates and vertebrates, despite the fact that the former typically have much larger population sizes than the latter [1]. It was argued that the non-recombining nature of mtDNA makes its diversity prone to be shaped by natural selection for new and beneficial variants - eradicating, or at least reducing, a correlation between population size and genetic variability. In addition, strong purifying selection against deleterious mtDNA mutations in the female germline could further homogenize levels of diversity (background selection) [2]. The observation that the fixation rate of non-synonymous substitutions in mtDNA correlates positively with $N_{e}$ in broad evolutionary comparisons [1] supports the idea that neutral variants frequently hitchhike along with positively selected alleles in the mtDNA genome when $N_{e}$ is large.

The report by Bazin and colleagues [1] sparked off debate, as did a study suggesting that mtDNA of birds would be particularly susceptible to selection, as it is in complete linkage disequilibrium with the maternally inherited $\mathrm{W}$ sex chromosome [3]. Birds have female heterogamety (males $\mathrm{ZZ}$, females $\mathrm{ZW}$ ), meaning that mothers transmit both mtDNA and the $\mathrm{W}$ chromosome to their daughters (Figure 1). Like the mammalian Y chromosome, the avian $\mathrm{W}$ chromosome only recombines in a small pseudoautosomal region. Theoretically, this would mean that selection in the non-recombining part of the $\mathrm{W}$ chromosome would also affect mtDNA, and vice versa. The $\mathrm{W}$ chromosome itself has very low within-species variability, at least in chickens, testifying to a strong role for selection [4]. Does this mean that selection on avian mtDNA masks the expected signal of $N_{e}$ on diversity and that diversity estimates in avian mtDNA are thus especially poor predictors of effective population size, potentially reducing the value of this marker in, for 


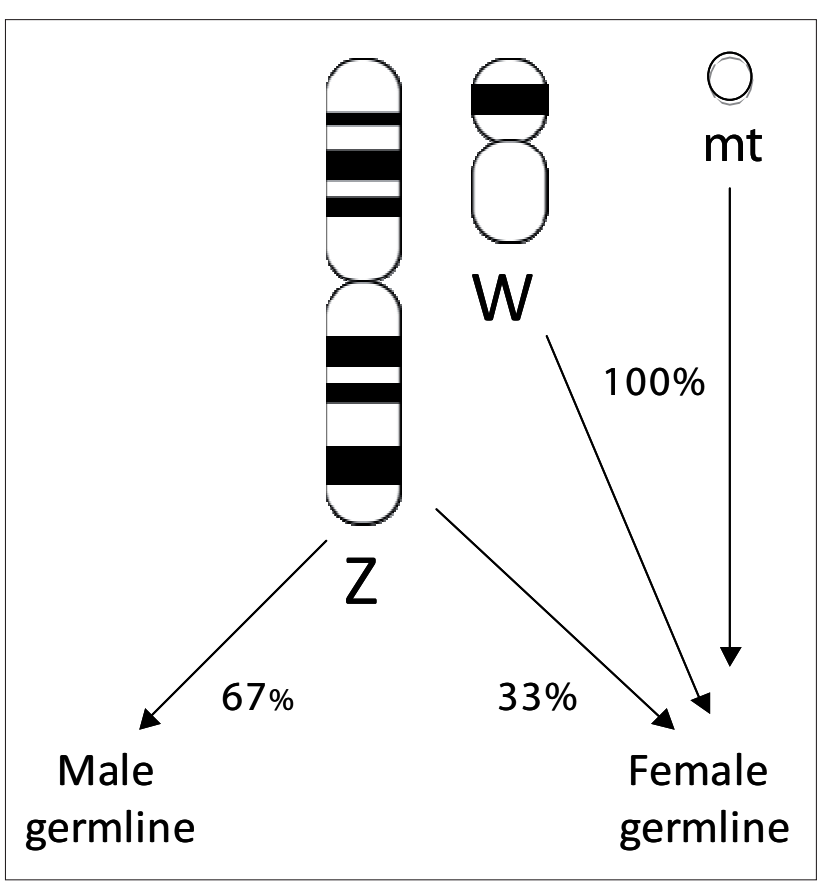

Figure I

Schematic illustration of the inheritance pattern of $m t D N A$ and sex chromosomes in birds and other female heterogametic systems.

example, biodiversity studies [5,6]? Rather, is the rate of mutation the main factor regulating mtDNA diversity in birds? Answers to these questions are now brought closer by a recent study by Nabholz et al. published in BMC Evolutionary Biology [7], which compares mtDNA divergence and diversity estimates for a large number of bird species with various types of life history, including different population structures and population sizes.

\section{Variation in avian mutation rates}

As the rate of mutation is difficult to measure directly, the number of substitutions that have accumulated during a certain time period in an evolutionary lineage is often taken as a proxy for the mutation rate. Nabholz et al. [7] gathered mtDNA sequence data from more than 1,000 bird species, available in GenBank, and used divergence estimates from these together with fossil calibration points to estimate substitution rates, and thereby, indirectly, mutation rates. The new data indicate that there is more than an order of magnitude variation in the mtDNA substitution rate among different bird lineages, challenging the ' $2 \%$-rule', which is yet another question under debate [8]. That idea stipulates that the mtDNA mutation rate follows a 'molecular clock' corresponding to $2 \%$ sequence divergence every million years of evolution (0.02 substitutions per site per million years). Nabholz et al. [7] find that substitution rate is positively correlated with metabolic rate and negatively correlated with longevity, two life-history traits previously suggested to explain deviations from a molecular clock.

The metabolic hypothesis posits that the production of mutagenic free radicals - reactive oxygen species (ROS), byproducts of respiration - increases with increasing rates of respiration, and so, therefore, does the rate of mutation. The longevity hypothesis proposes that long-lived organisms have evolved decreased rates of mtDNA mutation as an adaptation to reduce the deleterious effects of somatic mutations accumulating during their lifetime. Birds have higher metabolic rates than mammals, but when Nabholz et al. [7] compared the rates of mtDNA evolution in both groups, they found that avian mtDNA mutates at a significantly lower rate than that of mammals, especially when body size is regressed out of the analysis. They therefore favor life span as the main determinant of avian mtDNA mutation rates.

\section{The determinants of avian mtDNA diversity}

The significant heterogeneity in substitution rates observed in avian mtDNA from different species means that there is scope for variation in mutation rate to also explain variations in within-species mtDNA diversity. Indeed, Nabholz et al. [7] find that substitution rate and level of polymorphism are positively related. However, two proxys for $N_{e}$ (body size and current population census size) are not. These observations support the idea that avian mtDNA diversity is primarily due to the extent of mutational input, not to the effective population size, corroborating the previous claim based on data from other organisms [1].

Previously, the same authors had analyzed mammalian mtDNA for a correlation between the amount of adaptive evolution and $N_{e}$ but no correlation was found [9], which is at odds with the general idea that the efficiency of selection relates to life history. It may be that the relatively small populations typical of mammals mean that genetic drift plays a big role in producing mtDNA diversity but, nonetheless, $N_{e}$ does not seem to correlate with mtDNA diversity in mammals [9]. Perhaps past demographic changes or the influence of mutation rate variation mask a signal from present-day $N_{e}$. In their new study, Nabholz et al. [7] do not find a strong footprint of selection in avian mtDNA, similar to the situation in mammals [7]. Mutation rate variation therefore remains a vital explanation for variation in mtDNA diversity of birds.

Does this mean that it is time to rewrite those parts of population genetics textbooks that deal with predictions of 
polymorphism levels? Clearly, as far as mtDNA is concerned, there does seem to be a need for revision. However, a cautionary note should be sounded to acknowledge the difficulties in properly estimating $N_{e}$ by proxys such as body size or longevity. At present, it would be premature to state that the role of selection in shaping levels of mtDNA diversity is more pronounced in birds or other female heterogametic taxa than in male heterogametic systems, such as mammals.

Even if levels of diversity in mtDNA do not give us an unbiased picture of the effective size of avian populations, the use of mtDNA as a genetic marker is still warranted for other purposes, such as studies of phylogenetics and phylogeography. New high-throughput sequencing technologies might, however, lead to a shift in focus from population genetic studies based on mtDNA to population genomic approaches based on nuclear DNA, to estimate effective population size. Large-scale analyses of nuclear DNA can provide better and more direct estimates of $N_{e^{\prime}}$ thereby allowing more careful tests of the relationship between life history and population genetic and molecular evolutionary parameters.

\section{References}

I. Bazin E, Glemin S, Galtier N: Population size does not influence mitochondrial genetic diversity in animals. Science 2006, 3|2:570572.

2. Stewart JB, Freyer C, Elson JL, Larsson NG: Purifying selection of mtDNA and its implications for understanding evolution and mitochondrial disease. Nat Rev Genet 2008, [Epub ahead of print].

3. Berlin S, Tomaras D, Charlesworth B: Low mitochondrial variability in birds may indicate Hill-Robertson effects on the W chromosome. Heredity 2007, 99:389-396.

4. Berlin S, Ellegren $\mathrm{H}$ : Chicken W: a genetically uniform chromosome in a highly variable genome. Proc Natl Acad Sci USA 2004, 101:15967-15969.

5. Ballard JWO, Whitlock MC: The incomplete natural history of mitochondria. Mol Ecol 2004, 13:729-744.

6. Hurst GDD, Jiggins FM: Problems with mitochondrial DNA as a marker in population, phylogeographic and phylogenetic studies: the effects of inherited symbionts. Proc Biol Sci 2005, 272:15251534.

7. Nabholz B, Glémin S, Galtier N: The erratic mitochondrial clock: variation of mutation rate, not population size, affects mtDNA diversity across birds and mammals. BMC Evol Biol 2009, 9:54.

8. Pereira SL, Baker AJ: A mitogenomic timescale for birds detects variable phylogenetic rates of molecular evolution and refutes the standard molecular clock. Mol Biol Evol 2006, 23: I73 I-1740.

9. Nabholz B, Mauffrey JF, Bazin E, Galtier N, Glemin S: Determination of mitochondrial genetic diversity in mammals. Genetics 2008, |78:35I-36I. 